\title{
A VARIATIONAL PROBLEM OF PHASE TRANSITIONS FOR A TWO-PHASE ELASTIC MEDIUM WITH ZERO COEFFICIENT OF SURFACE TENSION
}

\author{
V. G. OSMOLOVSKII
}

Dedicated to Vasilǐ Mikhaǔlovich Babich

\begin{abstract}
The variational problem on the equilibrium of a two-phase elastic medium is given in an extended form and is compared with the standard setting. The lower semicontinuity of the energy functional in the extended formulation is studied, and an example is constructed where no equilibrium states exist for a special class of residual strain tensors. In the case of isotropic media, a method is described for finding equilibrium states in explicit form. The notion of temperatures of phase transitions is introduced, their existence is proved, and their properties are studied.
\end{abstract}

\section{$\S 1$. INTRODUCTION}

In determining strain densities for the energy of a homogeneous anisotropic elastic medium, certain coefficients $a_{i j k l}$ such that

$$
a_{i j k l}=a_{j i k l}=a_{k l i j}=a_{i j l k}, \quad a_{i j k l} \xi_{i j} \xi_{k l} \geq \nu|\xi|^{2}, \quad i, j, k, l=1, \ldots, m,
$$

are fixed; here $\nu>0, \xi \in R_{s}^{m \times m}$, where $R_{s}^{m \times m}$ is the space of symmetric $(m \times m)$ matrices, $|\xi|^{2}=\xi_{i j} \xi_{i j}$, and summation from 1 to $m$ over repeating indices is implied (physically, only the cases where $m=2$ or $m=3$ are of interest). With the help of these coefficients, the strain energy density $F(M)$ is given by the relation

$$
\begin{aligned}
F(M) & =a_{i j k l}\left(e_{i j}(M)-\zeta_{i j}\right)\left(e_{k l}(M)-\zeta_{k l}\right), \\
e_{i j}(M) & =\frac{M_{i j}+M_{j i}}{2}, \quad \zeta \in R_{s}^{m \times m}, M \in R^{m \times m},
\end{aligned}
$$

where $R^{m \times m}$ is the space of $(m \times m)$-matrices.

Let $\Omega$ be a bounded domain with Lipschitz boundary. Then the strain energy of an elastic medium that occupies this domain in the nondeformed state is determined by the relation

$$
I[u]=\int_{\Omega}\{F(\nabla u)+g \cdot u\} d x+\int_{\partial \Omega} f \cdot u d S .
$$

Here the vector-valued function $u(x)=\left(u^{1}(x), \ldots, u^{m}(x)\right), x \in \Omega$, is the displacement field, $g(x)=\left(g^{1}(x), \ldots, g^{m}(x)\right), x \in \Omega$, is the external volume force field, $f(x)=$ $\left(f^{1}(x), \ldots, f^{m}(x)\right), x \in \partial \Omega$, is the external boundary force field, and $(\nabla u)_{i j}=u_{x_{j}}^{i}$.

2010 Mathematics Subject Classification. Primary 74B05.

Key words and phrases. Free surfaces, nonconvex variational problems, phase transitions in continuum mechanics.

Supported by RFBR (grant no. 08-01-00748). 
In this case, $e(\nabla u)$ is the strain tensor and $\zeta$ is the residual strain tensor. We assume that $g \in L_{2}\left(\Omega, R^{m}\right), f \in L_{2}\left(\partial \Omega, R^{m}\right)$.

To describe the admissible displacement fields, we fix $u_{0} \in W_{2}^{1}\left(\Omega, R^{m}\right)$ and a measurable subset $\Gamma_{0} \subset \partial \Omega$. The role of the domain of the functional (1.3) will be played by the set

$$
\mathbb{X}=\left\{u \in W_{2}^{1}\left(\Omega, R^{m}\right):\left.\left(u-u_{0}\right)\right|_{\Gamma_{0}}=0\right\} .
$$

By an equilibrium state of an elastic medium we mean a solution of the variational problem

$$
I[\widehat{u}]=\inf _{u \in \mathbb{X}} I[u], \quad \widehat{u} \in \mathbb{X} .
$$

It is well known that if $\left|\Gamma_{0}\right|>0$ (in what follows the modulus of a set denotes its Lebesgue measure; in the case in question it is $(m-1)$-dimensional), then problem (1.5) is uniquely solvable by the definition of the equilibrium displacement field $\widehat{u}$. If $\left|\Gamma_{0}\right|=0$, a criterion of its solvability is the condition

$$
\begin{aligned}
& \int_{\Omega} g \cdot v d x+\int_{\partial \Omega} f \cdot v d S=0 \text { for all } v \in \mathcal{R}, \\
& \mathcal{R}=\left\{v(x): v(x)=B x+x_{0}, B \in R^{m \times m}, B^{*}=-B, x_{0} \in R^{m}\right\},
\end{aligned}
$$

under which problem (1.5) is uniquely solvable up to a summand belonging to $\mathcal{R}$.

Assume that we have two elastic media characterized by coefficients $a_{i j k l}^{ \pm}$and tensors $\zeta^{ \pm}$. Denoting their energy densities by $F^{ \pm}$, we fix a measurable characteristic function $\chi(x), x \in \Omega$, and assume that the elastic medium with the strain energy density $F^{+}$is placed at the points $x$ for which $\chi(x)=1$ and the medium with the density $F^{-}$ is placed at the remaining points. Then for the resulting contact problem, the energy functional takes the form

$$
I_{\chi}[u]=\int_{\Omega}\left(\chi F^{+}(\nabla u)+(1-\chi) F^{-}(\nabla u)+g \cdot u\right) d x+\int_{\partial \Omega} f \cdot u d S, \quad u \in \mathbb{X} .
$$

We emphasize that the function $\chi$ in (1.7) is fixed, being a parameter of the problem.

In this case, by an equilibrium state we mean a solution of the variational problem

$$
I_{\chi}[\widehat{u}]=\inf _{u \in \mathbb{X}} I_{\chi}[u], \quad \widehat{u} \in \mathbb{X},
$$

which is uniquely solvable if $\left|\Gamma_{0}\right| \neq 0$. A criterion of its solvability for $\left|\Gamma_{0}\right|=0$ is condition (1.6) under which it is uniquely solvable up to a summand belonging to the set $\mathcal{R}$.

There exist elastic media in which the crystal structure may change [1, depending on the temperature and internal stresses. We assume that only two kinds of crystal structure + or - may occur; let $F^{ \pm}$denote their strain energy densities. Such media are said to be two-phase. The location of media with densities $F^{ \pm}$is not known a priori. To decribe it, we fix the set

$$
\mathbb{Z}^{\prime}=\left\{\chi \in L_{\infty}(\Omega): \chi(x)=\chi^{2}(x) \text { almost everywhere in } \Omega\right\}
$$

of all measurable characteristic functions and define the strain energy functional of a two-phase elastic medium by the relation

$$
\begin{array}{r}
I_{0}[u, \chi, t]=\int_{\Omega}\left(\chi\left(F^{+}(\nabla u)+t\right)+(1-\chi) F^{-}(\nabla u)+g \cdot u\right) d x+\int_{\partial \Omega} f \cdot u d S, \\
u \in \mathbb{X}, \quad \chi \in \mathbb{Z}^{\prime},
\end{array}
$$

where $t$ is the temperature, which is assumed to be constant in the domain $\Omega$ and which plays the role of a parameter of the problem. 
By an equilibrium state for the energy functional of a two-phase elastic medium for a fixed value of the temperature $t$, we mean a solution of the variational problem

$$
I_{0}\left[\widehat{u}_{t}, \hat{\chi}_{t}, t\right]=\inf _{u \in \mathbb{X}, \chi \in \mathbb{Z}^{\prime}} I_{0}[u, \chi, t], \quad \widehat{u}_{t} \in \mathbb{X}, \quad \hat{\chi}_{t} \in \mathbb{Z}^{\prime} .
$$

The function $\widehat{u}_{t}$ determines an equilibrium displacement field, and the function $\hat{\chi}_{t}$ specifies the distribution of phases in an equilibrium state. An equilibrium state is said to be one-phase if $\hat{\chi}_{t} \equiv 0$ or $\hat{\chi}_{t} \equiv 1$, and two-phase otherwise.

The distinction of the functional (1.7) from (1.10) is not only in the occurrence of the temperature $t$ (which is not fundamental for (1.7)), but also in the fact that the function $\chi$ in (1.10) is not a parameter but an argument of the energy functional.

The functional (1.10) is viewed as having the right to exist (see [1]), but it does not give a complete description of the strain energy of a two-phase elastic medium, because it ignores the surface energy of the boundary where the phases meet. It is customary to assume that this energy is proportional to the area of that boundary. To give this area in terms of the function $\chi$, we consider the expression

$$
\int_{\Omega}|D \chi| \equiv \sup _{h \in C_{0}^{1}\left(\Omega, R^{m}\right),|h| \leq 1} \int_{\Omega} \chi \operatorname{div} h d x .
$$

If the right-hand side of (1.12) is finite, then we say that $\chi \in B V(\Omega)$. In the case of a smooth boundary separating the phases, the right-hand side of (1.12) coincides with the area of this boundary. For arbitrary functions $\chi$, the quantity (1.12) is a natural generalization of that area [2]. We set

$$
\mathbb{Z}=\mathbb{Z}^{\prime} \cap B V(\Omega)
$$

and define a new two-phase elastic medium energy functional (this time, it takes into account the surface energy of the boundary separating the phases) by the relation

$$
I[u, \chi, t, \sigma]=I_{0}[u, \chi, t]+\sigma \int_{\Omega}|D \chi| .
$$

Here, $\sigma>0$ is the so-called surface tension coefficient.

By an equilibrium state corresponding to the functional (1.14) for fixed $t$ and $\sigma$, we mean a solution of the variational problem

$$
I\left[\widehat{u}_{t, \sigma}, \widehat{\chi}_{t, \sigma}, t, \sigma\right]=\inf _{u \in \mathbb{X}, \chi \in \mathbb{Z}} I[u, \chi, t, \sigma], \quad \widehat{u}_{t, \sigma} \in \mathbb{X}, \hat{\chi}_{t, \sigma} \in \mathbb{Z} .
$$

The involvement of surface energy improves the mathematical properties of the energy functional. It turns out [3] that problem (1.15) is solvable for $\left|\Gamma_{0}\right|>0$, and that property (1.6) is a criterion of its solvability for $\left|\Gamma_{0}\right|=0$. A different situation arises with problem (1.11).

In the present paper, results describing the properties of problem (1.11) are given. In the first part of the paper $(\S 2)$, it is explained why problem (1.11) is "bad". In particular, an example where it has no solution is presented. In the second part of the paper (§3), it is explained why this problem is "good": for isotropic media, in a number of cases, not only solvability is proved, but an explicit form of the solutions is also given. In the third part $(\S 4)$, the notion of temperatures $t_{ \pm}$of phase transitions is introduced, their

existence is proved, and it is explained why problem (1.11) is "good" for $t \geq t_{+}$and $t \leq t_{-}$for arbitrary energy densities.

\section{§2. A CRITERION FOR THE LOWER SEMiCONTINUity OF THE ENERGY FUNCTIONAL}

Besides the sets (1.9) and (1.13), we define

$$
\mathbb{Z}^{\prime \prime}=\left\{\chi \in L_{\infty}: 0 \leq \chi(x) \leq 1 \text { almost everywhere in } \Omega\right\} .
$$


For fixed $t$, we consider the following variational problem for the functional (1.10):

$$
I_{0}\left[\widetilde{u}_{t}, \widetilde{\chi}_{t}, t\right]=\inf _{u \in \mathbb{X}, \chi \in \mathbb{Z}^{\prime \prime}} I[u, \chi, t], \quad \widetilde{u}_{t} \in \mathbb{X}, \quad \tilde{\chi} \in \mathbb{Z}^{\prime \prime} .
$$

The variational problem (2.2) admits the following physical interpretation: the substances of two phases may occur at each point of the domain $x \in \Omega$, and the function $\chi(x)$ describes the part of each phase at this point.

We denote by $\mu^{\prime}(t)$ and $\mu^{\prime \prime}(t)$ the right-hand sides of (1.11) and (2.2), respectively. It turns out 4 that these numbers satisfy the relation

$$
\mu^{\prime}(t)=\mu^{\prime \prime}(t)
$$

The following lemma shows that the extension of the domain of the functional (1.10) by replacing the set $\mathbb{Z}^{\prime}$ with $\mathbb{Z}^{\prime \prime}$ is indeed useful.

Lemma 2.1. Suppose the variational problem (2.2) is solvable for some $t$. Then the variational problem (1.11) is also solvable for this $t$.

Proof. Let $\widetilde{u}, \widetilde{\chi}$ be a solution of (2.2). Since

$$
\begin{aligned}
I_{0}[\widetilde{u}, \widetilde{\chi}, t]= & \int_{\Omega}\left(F^{-}(\nabla \widetilde{u})+g \cdot \widetilde{u}\right) d x+\int_{\partial \Omega} f \cdot \widetilde{u} d S \\
& +\int_{\Omega} \widetilde{\chi}\left(\left(F^{+}(\nabla \widetilde{u})-F^{-}(\nabla \widetilde{u})\right)+t\right) d x,
\end{aligned}
$$

we have

$$
I_{0}[\widetilde{u}, \widetilde{\chi}, t] \geq I_{0}[\widehat{u}, \hat{\chi}, t]
$$

where $\widehat{u}=\widetilde{u}$ and

$$
\begin{aligned}
\hat{\chi} & = \begin{cases}1 & \text { if } \Phi(x, t)<0, \\
0 & \text { if } \Phi(x, t) \geq 0,\end{cases} \\
\Phi(x, t) & =\left(F^{+}(\nabla \widetilde{u}(x))-F^{-}(\nabla \widetilde{u}(x))\right)+t .
\end{aligned}
$$

Since $\hat{\chi} \in \mathbb{Z}^{\prime}$, from $(2.2)$ and $(2.5)$ we obtain $\mu^{\prime \prime}(t)=I_{0}[\widetilde{u}, \widetilde{\chi}, t] \geq I_{0}[\widehat{u}, \widehat{\chi}, t] \geq \mu^{\prime}(t)$. Then, by (2.3), the pair $\widehat{u}, \hat{\chi}$ is a solution of (1.11).

Our aim in this section is to establish necessary and sufficient conditions for the relation

$$
\begin{gathered}
I_{0}[u, \chi, t] \leq \liminf _{n \rightarrow \infty} I_{0}\left[u_{n}, \chi_{n}, t\right], \quad u, u_{n} \in W_{2}^{1}\left(\Omega, R^{m}\right), \quad \chi, \chi_{n} \in \mathbb{Z}^{\prime \prime}, t \in R^{1}, \\
u_{n} \neg u \text { in } W_{2}^{1}\left(\Omega, R^{m}\right), \quad \chi_{n} \stackrel{*}{\rightarrow} \chi
\end{gathered}
$$

Recall that $\chi_{n} \stackrel{*}{\rightarrow} \chi$ means that

$$
\int_{\Omega} \chi_{n} \phi d x \rightarrow \int_{\Omega} \chi \phi d x \text { as } n \rightarrow \infty \text { for all } \phi \in L_{1}(\Omega) .
$$

Obviously, the set $\mathbb{Z}^{\prime \prime}$ is closed with respect to this convergence.

The condition (2.7) of lower semicontinuity serves as a basis for the proof of the solvability of problem (2.2), and also of problem (1.11), in view of Lemma 2.1 (see, e.g., [5]).

Obviously, relation (2.7) is equivalent to a similar relation for the functional

$$
J[u, \chi]=\int_{\Omega}\left\{\chi F^{+}(\nabla u)+(1-\chi) F^{-}(\nabla u)\right\} d x, \quad u \in W_{2}^{1}\left(\Omega, R^{m}\right), \quad \chi \in \mathbb{Z}^{\prime \prime} .
$$


Theorem 2.1. The inequality

$$
J[u, \chi] \leq \liminf _{n \rightarrow \infty} J\left[u_{n}, \chi_{n}\right], \quad u, u_{n} \in W_{2}^{1}\left(\Omega, R^{m}\right), \quad \chi, \chi_{n} \in \mathbb{Z}^{\prime \prime},
$$

is valid for all $u, \chi$ and any sequences $u_{n}, \chi_{n}$ such that

$$
u_{n} \rightarrow u \text { in } W_{2}^{1}\left(\Omega, R^{m}\right), \quad \chi_{n} \stackrel{*}{\rightarrow} \chi,
$$

if and only if

$$
a_{i j k l}^{+}=a_{i j k l}^{-}, \quad \zeta_{i j}^{+}=\zeta_{i j}^{-}, \quad i, j, k, l=1, \ldots, m .
$$

Proof. The sufficiency of conditions (2.11) is obvious because

$$
F^{+}(M)=F^{-}(M) \text { for all } M \in R^{m \times m}
$$

(this follows from (2.11)). To prove the "only if" part, we shall construct special sequences (2.10) so that inequality (2.9) will imply (2.11). In constructing such sequences, we follow the approach of [5] and its adaptation to problems on phase transitions [6]. We fix the unit cube

$$
K=(0,1)^{m} \subset R^{m} .
$$

Suppose we are given functions

$$
\phi \in C_{0}^{\infty}\left(K, R^{m}\right), \quad \psi \in L_{\infty}(K), \quad 0 \leq \psi(x) \leq 1 \text { almost everywhere in } K .
$$

Denote by $\mathcal{N}$ the set of all vectors in $R^{m}$ with integral coordinates. We represent the space $R^{m}$, up to a set of zero measure, as a union of cubes:

$$
R^{m}=\bigcup_{z_{j} \in \mathcal{N}} K_{z_{j}}, \quad K_{z_{j}}=K+z_{j}
$$

Let $\widetilde{\phi}$ and $\widetilde{\psi}$ be periodic extensions of the functions $\phi$ and $\psi$ from the cube $K$ to the space $R^{m}$ :

$$
\widetilde{\phi}\left(z+z_{j}\right)=\phi(z), \quad \widetilde{\psi}\left(z+z_{j}\right)=\psi(z) \text { for } z \in K .
$$

Let $x^{0} \in \Omega$, and let a positive number $l$ be so small that

$$
K\left(l, x^{0}\right)=\left\{x \in R^{m}: x_{i}^{0}<x_{i}<l\right\} \subset \Omega .
$$

Using functions (2.15) and the cube (2.16), we define sequences $\widetilde{\phi}_{n}$ and $\widetilde{\psi}_{n}$ by the relations

$$
\widetilde{\phi}_{n}(x)=\frac{l}{n} \widetilde{\phi}\left(\frac{x-x^{0}}{l} n\right), \quad \widetilde{\psi}_{n}(x)=\widetilde{\psi}\left(\frac{x-x^{0}}{l} n\right), \quad n=1,2, \ldots
$$

Then the functions

$$
\begin{gathered}
\phi_{n}(x)=\left\{\begin{array}{lll}
\widetilde{\phi}_{n}(x) & \text { if } & x \in K\left(l, x^{0}\right), \\
0 & \text { if } & x \in \Omega \backslash K\left(l, x^{0}\right),
\end{array}\right. \\
\chi_{n}(x)=\left\{\begin{array}{lll}
\widetilde{\psi}_{n}(x) & \text { if } & x \in K\left(l, x^{0}\right), \\
0 & \text { if } & x \in \Omega \backslash K\left(l, x^{0}\right),
\end{array}\right. \\
n=1,2, \ldots, \quad x \in \Omega,
\end{gathered}
$$

belong to the spaces $C_{0}^{\infty}\left(\Omega, R^{m}\right)$ and $\mathbb{Z}^{\prime \prime}$, respectively.

The role of $u_{n}$ in (2.10) will be played by the sequence

$$
u_{n}=M\left(x-x^{0}\right)+\phi_{n}(x), \quad M \in R^{m \times m},
$$

and the $\chi_{n}$ will be chosen as in (2.18).

Straightforward calculations show that

$$
u_{n} \neg u=M\left(x-x^{0}\right) \text { in the space } W_{2}^{1}\left(\Omega, R^{m}\right)
$$


and

$$
\chi_{n} \stackrel{*}{\rightarrow} \chi, \quad \chi(x)= \begin{cases}\int_{K} \psi(y) d y & \text { if } x \in K\left(l, x^{0}\right), \\ 0 & \text { if } x \in \Omega \backslash K\left(l, x^{0}\right) .\end{cases}
$$

By (2.18), conditions (2.9) for the resulting sequence (2.10) take the form

$$
\begin{aligned}
J\left[u_{n}, \chi_{n}\right]= & \int_{\Omega \backslash K\left(l, x^{0}\right)} F^{-}(M) d x \\
& +\int_{K\left(l, x^{0}\right)}\left\{\widetilde{\psi}_{n}(x) F^{+}\left(M+\nabla \widetilde{\phi}_{n}(x)\right)+\left(1-\widetilde{\psi}_{n}(x)\right) F^{-}\left(M+\nabla \widetilde{\phi}_{n}(x)\right)\right\} d x .
\end{aligned}
$$

A series of transformations and changes of coordinates lead to the relation

$$
\begin{gathered}
\int_{K\left(l . x^{0}\right)}\left\{\widetilde{\psi}_{n}(x) F^{+}\left(M+\nabla \widetilde{\phi}_{n}(x)\right)+\left(1-\widetilde{\psi}_{n}(x)\right) F^{-}\left(M+\nabla \widetilde{\phi}_{n}(x)\right)\right\} d x \\
=l^{m} \int_{K}\left\{\psi(x) F^{-}(M+\nabla \phi(x))+(1-\psi(x)) F^{-}(M+\nabla \phi(x))\right\} d x .
\end{gathered}
$$

Consequently,

$$
\begin{aligned}
\liminf _{n \rightarrow \infty} J\left[u_{n}, \chi_{n}\right]= & \int_{\Omega \backslash K\left(l, x^{0}\right)} F^{-}(M) d x \\
& +l^{m} \int_{K}\left\{\psi(x) F^{-}(M+\nabla \phi(x))+(1-\psi(x)) F^{-}(M+\nabla \phi(x))\right\} d x .
\end{aligned}
$$

Next, for the pair $u, \chi$ as in (2.20), (2.21) we have

$$
\begin{aligned}
J[u, \chi]= & \int_{\Omega}\left\{\chi F^{+}(\nabla u)+(1-\chi) F^{-}(\nabla u)\right\} d x=\int_{\Omega \backslash K\left(l, x^{0}\right)} F^{-}(M) d x \\
& +\int_{K\left(l, x^{0}\right)}\left\{\left(\int_{K} \psi(y) d y\right) F^{+}(M)+\left(1-\int_{K} \psi(y) d y\right) F^{-}(M)\right\} d x .
\end{aligned}
$$

Using (2.23) and (2.24), we write inequality (2.9) in the form

$$
\begin{aligned}
F^{+}(M) & \int_{K} \psi(x) d x+F^{-}(M)\left(1-\int_{K} \psi(x) d x\right) \\
\leq & \int_{K}\left\{\psi(x) F^{+}(M+\nabla \phi(x))+(1-\psi(x)) F^{-}(M+\nabla \phi(x))\right\} d x .
\end{aligned}
$$

Since

$$
F^{ \pm}(M+\nabla \phi(x))=F^{ \pm}(M)+F_{M_{i j}}^{ \pm}(M) \phi_{x_{j}}^{i}(x)+\frac{1}{2} F_{M_{i j} M_{k l}}^{ \pm} \phi_{x_{j}}^{i}(x) \phi_{x_{l}}^{k}(x),
$$

inequality (2.25) is equivalent to the relation

$$
\begin{aligned}
0 \leq & F_{M_{i j}}^{+}(M) \int_{K} \psi(x) \phi_{x_{j}}^{i}(x) d x+F_{M_{i j}}^{-}(M) \int_{K}(1-\psi(x)) \phi_{x_{j}}^{i}(x) d x \\
& +\frac{1}{2} F_{M_{i j} M_{k l}}^{+} \int_{K} \psi(x) \phi_{x_{j}}^{i}(x) \phi_{x_{l}}^{k}(x) d x+\frac{1}{2} F_{M_{i j} M_{k l}}^{-} \int_{K}(1-\psi(x)) \phi_{x_{j}}^{i}(x) \phi_{x_{l}}^{k}(x) d x .
\end{aligned}
$$

In this inequality we replace $\phi$ by $\epsilon \phi, \epsilon>0$, divide both sides of the inequality obtained by the positive number $\epsilon$, and let $\epsilon$ go to zero. We get

$$
0 \leq F_{M_{i j}}^{+}(M) \int_{K} \psi(x) \phi_{x_{j}}^{i}(x) d x+F_{M_{i j}}^{-}(M) \int_{K}(1-\psi(x)) \phi_{x_{j}}^{i}(x) d x .
$$


Since the function $\phi$ is compactly supported, this inequality is equivalent to

$$
0 \leq\left(F_{M_{i j}}^{+}(M)-F_{M_{i j}}^{-}(M)\right) \int_{K} \psi(x) \phi_{x_{j}}^{i}(x) d x .
$$

Since this must be true for both $\phi$ and $-\phi$, we obtain

$$
0=\left(F_{M_{i j}}^{+}(M)-F_{M_{i j}}^{-}(M)\right) \int_{K} \psi(x) \phi_{x_{j}}^{i}(x) d x .
$$

Therefore,

$$
\left(F_{M_{i j}}^{+}(M)-F_{M_{i j}}^{-}(M)\right) \phi_{x_{j}}^{i}(x) \equiv 0 \quad \text { for all } \quad \phi \in C_{0}^{\infty}(K), \quad M \in R^{m \times m} .
$$

Indeed, otherwise for the role of $\psi$ we can choose the characteristic function of the set of positivity or negativity of the left-hand side of (2.27), arriving at a contradiction with (2.26).

For each pair of vectors $\xi, \lambda \in R^{m}$, we take a function $\phi(x)$ such that

$$
\phi_{x_{j}}^{i}(\widehat{x})=\xi_{i} \lambda_{j}
$$

at some point $\widehat{x} \in K$. Then (2.27) implies that

$$
\left(F_{M_{i j}}^{+}(M)-F_{M_{i j}}^{-}(M)\right) \xi_{i} \lambda_{j}=0 \quad \text { for all } \quad M \in R^{m \times m}, \quad \lambda, \xi \in R^{m} .
$$

Consequently, $F_{M_{i j}}^{+}(M)=F_{M_{i j}}^{-}(M)$, i.e.,

$$
a_{i j k l}^{+}\left(M_{k l}-\zeta_{k l}^{+}\right)=a_{i j k l}^{-}\left(M_{k l}-\zeta_{k l}^{-}\right) .
$$

From (2.28) with $M_{k l}=\zeta_{k l}^{-}$, it follows that $a_{i j k l}^{+}\left(\zeta^{+}-\zeta^{-}\right){ }_{k l}=0$. Then, since (1.1) is positive definite, we have $\zeta^{+}=\zeta^{-}$. Relation (2.28) with $M=0$ shows that $a_{i j k l}^{+} \zeta_{k l}^{+}=$ $a_{i j k l}^{-} \zeta_{k l}^{-}$. Then $a_{i j k l}^{+} M_{k l}=a_{i j k l}^{-} M_{k l}$. Taking the matrix with zero entries (except for one of them) for $M$, we get $a_{i j k l}^{+}=a_{i j k l}^{-}$.

The absence of the weak lower semicontinuity of the energy functional in the case where conditions (2.11) fail, which is established by Theorem 2.1, may lead to the unsolvability of the variational problem (1.11). We give an example confirming this.

Example 2.1. An example where no equilibrium states exist will be constructed in several steps.

Construction of the energy functional. Consider the functional (1.10) with $\Gamma_{0}=\partial \Omega$, $u_{0}=0, t=0, g=0$, and $f=0$. We describe the residual strain tensors that we need. Let $N_{ \pm}=\left\{x \in R^{m}: \zeta^{ \pm} x=0\right\}$. We assume that

$$
N_{+}=N_{-} \equiv N, \quad \operatorname{dim} N=m-1 .
$$

We set

$$
l \in R^{m}, \quad|l|=1, \quad l \perp N .
$$

By the symmetry of the matrices $\zeta^{ \pm}$, we have $\zeta^{ \pm} l=\alpha_{ \pm} l, \alpha_{ \pm} \in R^{1}$. The matrices $\zeta^{ \pm}$ will be fixed by the condition

$$
\zeta^{+} l=l, \quad \zeta^{-} l=-l .
$$

Construction of a special family of displacement fields. Obviously, the functions

$$
u^{ \pm}(x)=\zeta^{ \pm} x-x_{0}^{ \pm}, \quad x, x_{0}^{ \pm} \in R^{m},
$$

satisfy the equations

$$
e\left(\nabla u^{ \pm}\right)=\zeta^{ \pm}
$$


Using the decomposition

$$
x=\widetilde{x}+s l, \quad \widetilde{x} \in N, \quad s \in R^{1},
$$

we write the functions $u^{ \pm}$in the form

$$
u^{ \pm}(x)= \pm s l-x_{0}^{ \pm} .
$$

Fix a number $\delta>0$. We choose the vectors $x_{0}^{ \pm}$in such a way that

$$
\begin{array}{ll}
u^{-}(x)=0 & \text { if } x \in\{N+2 \delta l\}, \quad u^{-}(x)=u^{+}(x) \text { if } x \in\{N+\delta l\}, \\
u^{+}(x)=0 & \text { if } x \in N .
\end{array}
$$

Conditions (2.36) are fulfilled for $x_{0}^{+}=0, x_{0}^{-}=-2 \delta l$. For such $x_{0}^{ \pm}$, the functions (2.35) have the form

$$
u^{+}(x)=s l, \quad u^{-}(x)=(2 \delta-s) l .
$$

For the vectors $x$ in (2.34), we consider the function

$$
u_{\delta}(x)= \begin{cases}0 & \text { if } s \leq 0, \\ s l & \text { if } s \in[0, \delta], \\ (2 \delta-s) l & \text { if } s \in[\delta, 2 \delta] \\ 0 & \text { if } s \geq 2 \delta .\end{cases}
$$

We split the space $R^{m}$ into the bands

$$
\begin{aligned}
\Pi_{k} & =\left\{x \in R^{m}: \text { the number } s \text { in }(2.34) \text { belongs to }[2 k \delta, 2(k+1) \delta]\right\}, \\
k & =\ldots,-2,-1,0,1,2, \ldots
\end{aligned}
$$

For each band $\Pi_{k}$, we define the function $u_{\delta}^{k}(x)=u_{\delta}(x+2 k \delta l)$. Then the function

$$
\widehat{u}_{\delta}(x)=\sum_{k=-\infty}^{\infty} u_{\delta}^{k}(x)
$$

satisfies

$$
\widehat{u}_{\delta} \in W_{2, \mathrm{loc}}^{1}\left(R^{m}, R^{m}\right), \quad\left|\widehat{u}_{\delta}(x)\right| \leq C \delta \quad \text { for all } \quad x \in R^{m} .
$$

Moreover, by (2.33),

$$
\begin{aligned}
e\left(\nabla \hat{u}_{\delta}\right) & =\hat{\chi}_{\delta} \zeta^{+}+\left(1-\hat{\chi}_{\delta}\right) \zeta^{-}, \\
\hat{\chi}_{\delta} & =\left\{\begin{array}{lll}
1 & \text { if } x \in \Pi_{k}^{+}, \Pi_{k}^{+}=\left\{x \in \Pi_{k}: s \in[2 k \delta, 2(k+1) \delta]\right\}, \\
0 & \text { if } x \in \Pi_{k}^{-}, \Pi_{k}^{-}=\left\{x \in \Pi_{k}: s \in((2 k+1) \delta, 2(k+1) \delta)\right\} .
\end{array}\right.
\end{aligned}
$$

Property (2.42) shows that

$$
I_{0}\left[\widehat{u}_{\delta}, \widehat{\chi}_{\delta}, 0\right]=0 \text {. }
$$

Calculation of the infimum of the energy functional. We prove that, in the case under consideration,

$$
\inf _{u \in \mathbb{X}, \chi \in \mathbb{Z}^{\prime}} I_{0}[u, \chi, 0]=0 .
$$

For all sufficiently small positive $\rho$, we consider a function $\phi_{\rho}$ such that

$$
\phi_{\rho} \in C_{0}^{\infty}(\Omega), \quad \phi_{\rho}(x)=1 \quad \text { if } \quad \operatorname{dist}(x, \partial \Omega)>\rho, \quad\left|\nabla \phi_{\rho}(x)\right| \leq \frac{C}{\rho} .
$$

Since

$$
e\left(\nabla\left(\phi_{\rho} \widehat{u}_{\delta}\right)\right)=\phi_{\rho} e\left(\nabla \widehat{u}_{\delta}\right)+e\left(\nabla \phi_{\rho} \otimes \widehat{u}_{\delta}\right), \quad\left(\nabla \phi_{\rho} \otimes \widehat{u}_{\delta}\right)_{i j}=\phi_{\rho x_{i}} \widehat{u}_{\delta}^{j},
$$


relations (2.42) and (2.45) yield

$$
\begin{aligned}
e\left(\nabla\left(\phi_{\rho} \widehat{u}_{\delta}\right)\right) & =\widehat{\chi}_{\delta} \zeta^{+}+\left(1-\hat{\chi}_{\delta}\right) \zeta^{-} \text {if } \operatorname{dist}(x, \partial \Omega)>\rho, \\
\left|e\left(\nabla\left(\phi_{\rho} \widehat{u}_{\delta}\right)\right)\right| & \leq C \frac{\delta}{\rho} \text { if } \operatorname{dist}(x, \partial \Omega) \leq \rho .
\end{aligned}
$$

Therefore,

$$
\begin{aligned}
& \hat{\chi}_{\delta} F^{+}\left(\nabla\left(\phi_{\rho} \widehat{u}_{\delta}\right)\right)+\left(1-\hat{\chi}_{\delta}\right) F^{-}\left(\nabla\left(\phi_{\rho} \widehat{u}_{\delta}\right)\right)=0 \text { if } \operatorname{dist}(x, \partial \Omega)>\rho, \\
& \hat{\chi}_{\delta} F^{+}\left(\nabla\left(\phi_{\rho} \widehat{u}_{\delta}\right)\right)+\left(1-\hat{\chi}_{\delta}\right) F^{-}\left(\nabla\left(\phi_{\rho} \widehat{u}_{\delta}\right)\right) \leq C\left(\frac{\delta}{\rho}+1\right)^{2} \text { if } \operatorname{dist}(x, \partial \Omega) \leq \rho .
\end{aligned}
$$

Thus,

$$
I_{0}\left[\widehat{u}_{\delta} \phi_{\rho}, \hat{\chi}_{\delta}, 0\right] \leq C\left(\frac{\delta}{\rho}+1\right)^{2} \delta .
$$

Putting $\rho=\delta$ in this inequality and using the fact that $\widehat{u}_{\delta} \phi_{\rho} \in \mathbb{X}$ and the nonnegativity of the functional $I_{0}[u, \chi, 0]$, we see that $(2.44)$ is valid.

Proof of the unsolvability of the variational problem (1.5) for the resulting energy functional. If the functional $I_{0}[u, \chi, 0]$ has an equilibrium state $\widehat{u}, \hat{\chi}$, then, by $(2.44)$,

$$
e(\nabla \widehat{u})=\hat{\chi} \zeta^{+}+(1-\hat{\chi}) \zeta^{-}, \quad \widehat{u} \in \stackrel{\circ}{W_{2}^{1}}\left(\Omega, R^{m}\right), \quad \hat{\chi} \in \mathbb{Z}^{\prime} .
$$

Conversely, any solution $\widehat{u}, \hat{\chi}$ of problem (2.47) is an equilibrium state for the functional $I_{0}[u, \chi, 0]$. We prove that for the tensors $\zeta^{ \pm}$satisfying conditions $(2.29)$ and $(2.31)$, problem (2.47) has no solution.

Transforming (2.47), we obtain

$$
\widehat{u}_{x_{j}}^{i}+\widehat{u}_{x_{i}}^{j}=2\left(\widehat{\chi} \zeta_{i j}^{+}+(1-\hat{\chi}) \zeta_{i j}^{-}\right)
$$

Let $\tau$ be a unit vector of the subspace $N$. Multiplying the two sides of (2.48) by $\tau_{i} \tau_{j}$, $\tau_{i} l_{j}$, and $l_{i} l_{j}$ and summing over repeating indices, we arrive at the relations

$$
\frac{\partial(\widehat{u} \cdot \tau)}{\partial \tau}=0, \quad \frac{\partial(\widehat{u} \cdot \tau)}{\partial l}+\frac{\partial(\widehat{u} \cdot l)}{\partial \tau}=0, \quad \frac{\partial(\widehat{u} \cdot l)}{\partial l}=2 \widehat{\chi}-1
$$

Fixing $\tau \in N$, we denote by $M$ the subspace of $R^{m}$ orthogonal to this vector. It is obvious that $l \in M$. We set

$$
\Omega_{x}=\left\{r \in R^{1}: \Omega \cap\{x+r \tau\}\right\}, \quad x \in M .
$$

Since $\Omega_{x}$ is an open set on a line, either it is empty or it is a union of an at most countable collection of open disjoint intervals $d_{j}$.

The function $\widehat{u}$ belongs to $\mathbb{X}=\stackrel{\circ}{W}_{2}^{1}\left(\Omega, R^{m}\right)$. Consequently, for almost all $x \in M$ with $\Omega_{x} \neq \varnothing$, the restriction of the function $\widehat{u} \cdot \tau$ to the interval $d_{j}$ lies in the space $\stackrel{\circ}{W}{ }_{2}^{1}\left(d_{j}\right)$ for each $j$, and the Sobolev derivative with respect to $r \in d_{j}$ of this restriction coincides with the restriction of the Sobolev derivative $\partial \widehat{u} \cdot \tau / \partial \tau$ to the interval $d_{j}$.

From the above arguments and the first relation in (2.49), it follows that $\widehat{u} \cdot \tau=0$ on a set $E_{\tau} \subset \Omega$ of full measure. Then, for the same reasons, the second relation in (2.49) shows that $\widehat{u} \cdot l=0$ on a set $E_{\tau}^{\prime} \subset \Omega$ of full measure. Repeating these arguments for the elements of a basis $\tau^{1}, \ldots, \tau^{m-1}$ of the space $N$, we conclude that $\widehat{u}=0$ almost everywhere in $\Omega$, but this contradicts the third equation in (2.49).

The construction of the above example is based on the techniques developed in [7, 8]. The fact that any solution of (1.11) solves (2.2) enables us to state the following necessary minimum condition. 
Lemma 2.2. Let a pair $\widehat{u}, \hat{\chi}$ be a solution of problem (1.11). Then

$$
\begin{aligned}
& \int_{\Omega}\left\{\left(\widehat{\chi} F_{M_{i j}}^{+}(\nabla \widehat{u})+(1-\hat{\chi}) F_{M_{i j}}^{-}(\nabla \widehat{u})\right) v_{x_{j}}^{i}\right\} d x \\
& \quad+\int_{\partial \Omega} f \cdot v d S=0 \quad \text { for all } v \in W_{2}^{1}\left(\Omega, R^{m}\right),\left.v\right|_{\Gamma_{0}}=0, \\
& \left(F^{+}(\nabla \widehat{u})-F^{-}(\nabla \widehat{u})\right)+t \leq 0 \quad \text { for a.e. } x \in \Omega \text { with } \hat{\chi}(x)=1, \\
& \left(F^{+}(\nabla \widehat{u})-F^{-}(\nabla \widehat{u})\right)+t \geq 0 \quad \text { for a.e. } x \in \Omega \text { with } \hat{\chi}(x)=0 .
\end{aligned}
$$

Proof. Note that for all $s \in[0,1]$ and all $\chi \in \mathbb{Z}^{\prime \prime}$, if $v$ is as in the lemma, then

$$
\widehat{u}+s v \in \mathbb{X}, \quad(1-s) \hat{\chi}+s \chi \in \mathbb{Z}^{\prime \prime} .
$$

Since the pair $\widehat{u}, \hat{\chi}$ is a solution of problem (2.2), the function

$$
\phi(s)=I_{0}[\widehat{u}+s v,(1-s) \hat{\chi}+s \chi, t]
$$

attains its minimal value for $s=0$. Consequently,

$$
\phi^{\prime}(0) \geq 0
$$

Expanding this inequality, we obtain

$$
\begin{gathered}
\int_{\Omega}\left(\hat{\chi} F_{M_{i j}}^{+}(\nabla \widehat{u})+(1-\hat{\chi}) F_{M_{i j}}^{-}(\nabla \widehat{u})\right) v_{x_{j}}^{i} d x+\int_{\partial \Omega} f \cdot v d S \\
+\int_{\Omega}(\chi-\hat{\chi})\left(\left(F^{+}(\nabla \widehat{u})-F^{-}(\nabla \widehat{u})\right)+t\right) d x \geq 0 .
\end{gathered}
$$

Obviously, this is equivalent to (2.50).

\section{§3. ISOTROPIC TWO-PHASE MEDIA}

The absence of the lower semicontinuity of the energy functional (see Theorem 2.1) may (see Example 2.1), or may not, lead to the unsolvability of the variational problem (1.11). Our aim in this section is to describe a method that enables us not only to prove the existence of equilibrium states in some cases but also to derive explicit formulas for the functions $\widehat{u}$ and $\hat{\chi}$.

This method works in the case of isotropic media,

$$
a_{i j k l}^{ \pm}=\frac{a_{ \pm}}{2}\left(\delta_{i k} \delta_{j l}+\delta_{i l} \delta_{j k}\right)+b_{ \pm} \delta_{i j} \delta_{k l}, \quad a_{ \pm}>0, \quad b_{ \pm} \geq 0, \quad \zeta_{i j}^{ \pm}=c_{ \pm} \delta_{i j}, \quad c_{ \pm} \in R^{1},
$$

under the additional condition that the strain energy densities $F^{ \pm}(M)$ are close:

$$
a_{+}=a_{-} \equiv a \text {. }
$$

Note that in the case of (3.1),

$$
\begin{array}{r}
F^{ \pm}(M)=a_{ \pm} \operatorname{tr}\left(e(M)-c_{ \pm} i\right)^{2}+b_{ \pm} \operatorname{tr}^{2}\left(e(M)-c_{ \pm} i\right), \\
\text { where } i \text { is the identity matrix in the space } R^{m} .
\end{array}
$$

The main idea of the method is based on the following statement 9 . 
For the energy functional $I_{0}[u, \chi, t]$ with densities (3.3), for all $u \in W_{2}^{1}\left(\Omega, R^{m}\right)$, all $\chi \in \mathbb{Z}^{\prime}$, and all $t \in R^{1}$, in the case of zero force fields $g$ and $f$ we have

$$
\begin{aligned}
& I_{0}[u, \chi, t]=G(Q, t, \nu)+\int_{\Omega} \frac{a_{+} \chi+a_{-}(1-\chi)}{4}|\operatorname{curl} u|^{2} d x \\
& +\int_{\Omega}\left(a_{+} \chi+a_{-}(1-\chi)\right)\left(u_{x_{j}}^{i} u_{x_{i}}^{j}-u_{x_{i}}^{i} u_{x_{j}}^{j}\right) d x \\
& +\int_{\Omega}\left(\left(a_{+}+b_{+}\right) \chi+\left(a_{-}+b_{-}\right)(1-\chi)\right) \\
& \times\left(\operatorname{div} u-\frac{c_{+}\left(a_{+}+b_{+} m\right)-\frac{\lambda}{2}}{a_{+}+b_{+}} \chi-\frac{c_{-}\left(a_{-}+b_{-} m\right)-\frac{\lambda}{2}}{a_{-}+b_{-}}(1-\chi)\right)^{2} d x, \\
& (\operatorname{curl} u)_{i j}=u_{x_{j}}^{i}-u_{x_{i}}^{j} \text {, } \\
& |\Omega|^{-1} G(Q, t, \nu)=t Q-\nu \lambda+\left(m c_{+}^{2}\left(a_{+}+b_{+} m\right)-\frac{\left(c_{+}\left(a_{+}+b_{+} m\right)-\frac{\lambda}{2}\right)^{2}}{a_{+}+b_{+}}\right) Q \\
& +\left(m c_{-}^{2}\left(a_{-}+b_{-} m\right)-\frac{\left(c_{-}\left(a_{-}+b_{-} m\right)-\frac{\lambda}{2}\right)^{2}}{a_{-}+b_{-}}\right)(1-Q), \\
& Q=\frac{1}{|\Omega|} \int_{\Omega} \chi d x, \quad \nu=\frac{1}{|\Omega|} \int_{\partial \Omega} u_{n} d S, \\
& \lambda=\lambda(Q, \nu)=2 \frac{\frac{c_{+}\left(a_{+}+b_{+} m\right)}{a_{+}+b_{+}} Q+\frac{c_{-}\left(a_{-}+b_{-} m\right)}{a_{-}+b_{-}}(1-Q)}{\frac{Q}{a_{+}+b_{+}}+\frac{1-Q}{a_{-}+b_{-}}}-\frac{2}{\frac{Q}{a_{+}+b_{+}}+\frac{1-Q}{a_{-}+b_{-}}} \nu .
\end{aligned}
$$

We consider the auxiliary functional

$$
\begin{aligned}
J[u, \chi, t, \nu]= & J_{0}[u, \chi, t]+G(Q, t, \nu), \\
J_{0}[u, \chi, t]= & \int_{\Omega} \frac{a_{+} \chi+a_{-}(1-\chi)}{4}|\operatorname{curl} u|^{2} d x \\
& +\int_{\Omega}\left(\left(a_{+}+b_{+}\right) \chi+\left(a_{-}+b_{-}\right)(1-\chi)\right) \\
& \times\left(\operatorname{div} u-\frac{c_{+}\left(a_{+}+b_{+} m\right)-\frac{\lambda}{2}}{a_{+}+b_{+}} \chi-\frac{c_{-}\left(a_{-}+b_{-} m\right)-\frac{\lambda}{2}}{a_{-}+b_{-}}(1-\chi)\right)^{2} d x .
\end{aligned}
$$

The functional (3.5) differs from the initial energy functional by the absence of the third summand on the right-hand side in (3.4).

Let $\mathbb{X}^{\nu}$ be a subset of functions in $W_{2}^{1}\left(\Omega, R^{m}\right)$ with fixed $\nu$ (see (3.4)). For fixed $t$ and $\nu$, we consider the variational problem

$$
J\left[\widehat{u}_{t, \nu}, \hat{\chi}_{t, \nu}, t, \nu\right]=\inf _{u \in \mathbb{X}^{\nu}, \chi \in \mathbb{Z}^{\prime}} J[u, \chi, t, \nu], \quad \widehat{u}_{t, \nu} \in \mathbb{X}^{\nu}, \hat{\chi}_{t, \nu} \in \mathbb{Z}^{\prime} .
$$

To study this problem, we shall use a solution $\widehat{Q}_{t, \nu} \in[0,1]$ of the extremum problem

$$
G\left(\widehat{Q}_{t, \nu}, t, \nu\right)=\inf _{Q \in[0,1]} G(Q, t, \nu),
$$

which exists necessarily by the continuity of the function $G(., t, \nu)$ on the interval $[0,1]$.

We fix numbers $t$ and $\nu$. Let $\widehat{Q}_{t, \nu}$ be a solution of problem (3.7). Suppose $\widetilde{\chi}_{t, \nu} \in \mathbb{Z}^{\prime}$ is a function satisfying

$$
\widehat{Q}_{t, \nu}=\frac{1}{|\Omega|} \int_{\Omega} \tilde{\chi}_{t, \nu} d x
$$


and such that the system of equations

$$
\begin{aligned}
\operatorname{curl} u & =0, \\
\operatorname{div} u & =\frac{c_{+}\left(a_{+}+b_{+} m\right)-\frac{\lambda}{2}}{a_{+}+b_{+}} \chi+\frac{c_{-}\left(a_{-}+b_{-} m\right)-\frac{\lambda}{2}}{a_{-}+b_{-}}(1-\chi), \quad \lambda=\lambda\left(\widehat{Q}_{t, \nu}, \nu\right),
\end{aligned}
$$

admits a solution $u=\widetilde{u}_{t, \nu} \in \mathbb{X}^{\nu}$ (the necessary solvability condition for the second equation of this system in the space $\mathbb{X}^{\nu}$ is fulfilled by the choice of the number $\lambda$ ). Obviously, then the variational problem (3.6) is solvable and the set of its solutions consists only of the pairs $\widehat{u}_{t, \nu}=\widetilde{u}_{t, \nu}, \hat{\chi}_{t, \nu}=\widetilde{\chi}_{t, \nu}$.

To describe the set of all solutions of problem (3.7), we define functions $t_{ \pm}(\nu)$ by the relations

$$
\begin{aligned}
t_{+}(\nu)= & -\nu^{2}[a+b] \frac{a_{-}+b_{-}}{a_{+}+b_{+}} \\
& +2 \nu[c(a+b m)] \frac{a_{-}+b_{-}}{a_{+}+b_{+}}+\frac{[c(a+b m)]^{2}}{a_{+}+b_{+}}-\left[m c^{2}(a+b m)\right], \\
t_{-}(\nu)= & -\nu^{2}[a+b] \frac{a_{+}+b_{+}}{a_{-}+b_{-}} \\
& +2 \nu[c(a+b m)] \frac{a_{+}+b_{+}}{a_{-}+b_{-}}-\frac{[c(a+b m)]^{2}}{a_{-}+b_{-}}-\left[m c^{2}(a+b m)\right] .
\end{aligned}
$$

Here the square brackets denote the jump of the quantity enclosed: $[\alpha]=\alpha_{+}-\alpha_{-}$. Obviously,

$$
t_{+}(\nu)-t_{-}(\nu)=([c(a+b m)]-\nu[a+b])^{2} \frac{\left(a_{+}+b_{+}\right)+\left(a_{-}+b_{-}\right)}{\left(a_{+}+b_{+}\right)\left(a_{-}+b_{-}\right)} \geq 0 .
$$

We split the plane of parameters $\{\nu, t\}$ into the following zones:

$$
\begin{aligned}
& V_{\geq}=\left\{\nu, t \in R^{1}: t \geq t_{+}(\nu)>t_{-}(\nu) \text { and } t>t_{+}(\nu)=t_{-}(\nu)\right\}, \\
& V_{\leq}=\left\{\nu, t \in R^{1}: t \leq t_{-}(\nu)<t_{+}(\nu) \text { and } t<t_{-}(\nu)=t_{+}(\nu)\right\}, \\
& V_{=}=\left\{\nu, t \in R^{1}: t=t_{+}(\nu)=t_{-}(\nu)\right\} \\
& V_{0}=\left\{\nu, t \in R^{1}: t \in\left(t_{-}(\nu), t_{+}(\nu)\right) \neq \varnothing\right\} .
\end{aligned}
$$

The set of all solutions $\hat{Q}_{t, \nu}$ of problem (3.7) was described in [9]; generally speaking, it is a multivalued function of the parameters $\{t, \nu\} \in R^{2}$.

The function $\widehat{Q}_{t, \nu}$ of parameters $\{t, \nu\} \in R^{2}$ possesses the following properties:

(a) the function $\hat{Q}_{t, \nu}$ is single-valued and continuous for $\{t, \nu\} \in R^{2} \backslash V_{=}$;

(b) we have

$$
\widehat{Q}_{t, \nu}= \begin{cases}0 & \text { if }\{t, \nu\} \in V_{\geq}, \\ 1 & \text { if }\{t, \nu\} \in V_{\leq}, \\ \text {any number in the interval }[0,1] & \text { if }\{t, \nu\} \in V_{=}\end{cases}
$$

(c) the function $\widehat{Q}_{t, \nu}$ is single-valued and infinitely differentiable on the set $V_{0}$; its first derivatives on this set are given by the formulas

$$
\begin{aligned}
\frac{\partial \hat{Q}_{t, \nu}}{\partial t} & =-\frac{1}{2}\left(\frac{\widehat{Q}_{t, \nu}}{a_{+}+b_{+}}+\frac{1-\widehat{Q}_{t, \nu}}{a_{-}+b_{-}}\right)^{3} \frac{\left(a_{+}+b_{+}\right)^{2}\left(a_{-}+b_{-}\right)^{2}}{[c(a+b m)-\nu(a+b)]^{2}}, \\
\frac{\partial \hat{Q}_{t, \nu}}{\partial \nu} & =\left(\frac{\widehat{Q}_{t, \nu}}{a_{+}+b_{+}}+\frac{1-\widehat{Q}_{t, \nu}}{a_{-}+b_{-}}\right) \frac{\left(a_{+}+b_{+}\right)\left(a_{-}+b_{-}\right)}{[c(a+b m)-\nu(a+b)]} .
\end{aligned}
$$


Now we turn to the variational problem (3.6). We fix a function

$$
p \in W_{2}^{2}\left(\Omega, R^{m}\right), \quad \Delta p \equiv \nu,
$$

and use this function to specify the set $\mathbb{X}^{\nu}$ occurring in (3.6): we set

$$
\mathbb{X}^{\nu} \subset \mathbb{Y}=\left\{u \in W_{2}^{1}\left(\Omega, R^{m}\right): u=v+\nabla p,\left.v_{n}\right|_{\partial \Omega}=0\right\} .
$$

Since for the functions $u$ as above we have

$$
\int_{\partial \Omega} u_{n} d S=\int_{\partial \Omega} \frac{\partial p}{\partial n} d S=\int_{\Omega} \Delta p d x=|\Omega| \nu,
$$

these functions form a subset of the space $W_{2}^{1}\left(\Omega, R^{m}\right)$ with a fixed $\nu$ (see (3.4)).

We replace the set of admissible displacement fields $\mathbb{X}$ in the variational problem (1.11) by $\mathbb{X}^{\nu}$. This yields the variational problem

$$
I_{0}\left[\widehat{u}_{t, \nu}, \hat{\chi}_{t, \nu}, t\right]=\inf _{u \in \mathbb{X}^{\nu}, \chi \in \mathbb{Z}^{\prime}} I_{0}[u, \chi, t], \quad \widehat{u}_{t, \nu} \in \mathbb{X}^{\nu}, \hat{\chi}_{t, \nu} \in \mathbb{Z}^{\prime}
$$

Note that, in terms of the function $v$, for the functions $u$ as in (3.16), system (3.9) has the form

$$
\begin{aligned}
\operatorname{curl} v & =0, \quad \operatorname{div} v=\alpha(Q, \nu)(\chi-Q),\left.\quad v_{n}\right|_{\partial \Omega}=0, \\
\alpha(Q, \nu) & =\frac{[c(a+b m)-\nu(a+b)]}{\left(a_{-}+b_{-}\right) Q+\left(a_{+}+b_{+}\right)(1-Q)} .
\end{aligned}
$$

In (3.18), only the boundary conditions dictated by the definition of the set $\mathbb{Y}$ are presented. Additional boundary conditions arise when the set $\mathbb{X}^{\nu}$ is given explicitly.

Since under condition (3.2), for the domains $\Omega$ with sufficiently smooth boundaries we have

$$
\begin{gathered}
\int_{\Omega}\left(a_{+} \chi+a_{-}(1-\chi)\right)\left(u_{x_{j}}^{i} u_{x_{i}}^{j}-u_{x_{i}}^{i} u_{x_{j}}^{j}\right) d x=a \int_{\Omega}\left(u_{x_{j}}^{i} u_{x_{i}}^{j}-u_{x_{i}}^{i} u_{x_{j}}^{j}\right) d x \\
=\int_{\Omega}\left(u_{n}^{2} \delta_{j} n_{j}-2 u_{n} \delta_{i} u^{i}-u^{i} u^{j} \delta_{j} n_{i}\right) d S, \quad \delta_{i}=\frac{\partial}{\partial x_{i}}-n_{i} n_{k} \frac{\partial}{\partial x_{k}},
\end{gathered}
$$

it follows that, in some cases, for $u \in \mathbb{X}^{\nu}$ the right-hand side of (3.19) is fully determined by the function $p$. In these cases, the variational problem (3.17) reduces to the variational problem (3.6) for the functional (3.5). Appropriate examples and the construction of solutions of system (3.18) for them were given in [9]. For some examples, the set $\mathbb{X}^{\nu}$ has structure (1.4), and in other examples it contains the slipping condition.

Thus, we obtain the following description of the set of all solutions of problem (3.17).

Theorem 3.1. Suppose that the right-hand side of (3.19) with functions in the set (3.16) is uniquely determined by the function $p$, and that system (3.18) is solvable in the class $\mathbb{X}^{\nu}$. Then, for each pair of parameters $t$ and $\nu$,

(a) in the set $V_{\geq}$, there is a unique equilibrium state $\widehat{u}=\nabla p, \hat{\chi}=0$;

(b) in the set $V_{\leq}$, there is a unique equilibrium state $\widehat{u}=\nabla p, \hat{\chi}=1$;

(c) in the set $V_{=}$, there are infinitely many distinct equilibrium states $\widehat{u}, \hat{\chi}$; for each of them we have $\widehat{u}=\nabla p$, and $\hat{\chi}$ is an arbitrary function belonging to $\mathbb{Z}^{\prime}$;

(d) in the set $V_{0}$, generally speaking, the number of equilibrium states is more than one, all equilibrium states are two-phase, and the volume part of each of the phases is uniquely determined by the parameters $t$ and $\nu$. 


\section{§4. TEMPERATURES OF PHASE TRANSITIONS}

The formula for the functional $I_{0}[u, \chi, t]$ suggests that, plausibly, there exist two numbers $t_{ \pm}$such that $-\infty<t_{-} \leq t_{+}<\infty$ and

for $t<t_{-}$, only equilibrium states $\widehat{u}=\widehat{u}^{+}, \widehat{\chi}=\chi^{+} \equiv 1$ exist;

for $t>t_{+}$, only equilibrium states $\widehat{u}=\widehat{u}^{-}, \hat{\chi}=\chi^{-} \equiv 0$ exist;

for $t_{-}<t_{+}$and $t \in\left(t_{-}, t_{+}\right)$no one-phase equilibrium states exist,

where the $\widehat{u}^{ \pm}$are the minimizers of the functionals

$$
I^{ \pm}[u]=\int_{\Omega}\left(F^{ \pm}(\nabla u)+g \cdot u\right) d x+\int_{\partial \Omega} f \cdot u d S, \quad u \in \mathbb{X},
$$

respectively.

The numbers $t_{ \pm}$(if they exist) are called the upper and lower temperatures of phase transitions. In (4.1), the existence of equilibrium states for $t<t_{-}$and $t>t_{+}$is claimed, but their existence for $t \in\left(t_{-}, t_{+}\right)$is not stated.

The following is a sufficient condition for the existence of the temperatures of phase transitions; see [10].

Let $g \in L_{\infty}\left(\Omega, R^{m}\right)$. Then the condition $\widehat{u}^{ \pm} \in W_{\infty}^{1}\left(\Omega, R^{m}\right)$ is sufficient for the existence of the temperatures $t_{ \pm}$for the sign + and the sign - , respectively.

If the temperatures $t_{ \pm}$exist, then for $t=t_{ \pm}$the energy functional has (possibly, not unique) equilibrium states $\widehat{u}^{ \pm}, \chi^{ \pm}$; see [10]. Next from (2.50) we obtain necessary conditions for the existence of the temperatures $t_{ \pm}$.

If the temperature $t_{+}$or $t_{-}$exists, then the following inequalities are fulfilled for almost all $x \in \Omega$ (respectively):

$$
\begin{aligned}
& \left(F^{+}\left(\nabla \hat{u}^{+}(x)\right)-F^{-}\left(\nabla \hat{u}^{+}(x)\right)\right)+t_{-} \leq 0, \\
& \left(F^{+}\left(\nabla \hat{u}^{-}(x)\right)-F^{-}\left(\nabla \hat{u}^{-}(x)\right)\right)+t_{+} \geq 0 .
\end{aligned}
$$

Example 4.1. Inequalities (4.3) make it possible to construct examples of the energy densities $F^{ \pm}(M)$ and the force field $g$ for which the temperatures $t_{ \pm}$do not exist.

We fix an energy density $F^{+}$and a function $\widehat{u}^{+}$with the properties

$$
\widehat{u}^{+} \in \stackrel{\circ}{W}_{2}^{1}\left(\Omega, R^{m}\right) \cap W_{2}^{2}\left(\Omega, R^{m}\right), \quad F^{+}\left(\hat{\nabla} u^{+}\right) \notin L_{\infty}(\Omega) .
$$

We define a function $g \in L_{2}\left(\Omega, R^{m}\right)$ by the relation

$$
g^{k}=a_{i j k l}^{+} \widehat{u}_{x_{j} x_{l}}^{+i} .
$$

Obviously, $\widehat{u}^{+}$is an equilibrium state of the functional $I^{+}[u]$ for $\Gamma_{0}=\partial \Omega$ and $u_{0}=0$.

We set

$$
a_{i j k l}^{-}=\frac{1}{2} a_{i j k l}^{+}, \quad \zeta_{i j}^{-}=\zeta_{i j}^{+} .
$$

In this case, the function

$$
F^{+}\left(\hat{\nabla} u^{+}(x)\right)-F^{-}\left(\nabla \hat{u}^{+}(x)\right)=\frac{1}{2} F^{+}\left(\nabla \widehat{u}^{+}(x)\right)
$$

is nonnegative and does not belong to the space $L_{\infty}(\Omega)$, which implies that the temperature $t_{-}$does not exist. An example of the nonexistence of the temperature $t_{+}$is constructed similarly. 
In the examples in $\S 3$, the temperatures of phase transitions exist and are given by the explicit formulas (3.10). In these examples, $\widehat{u}^{ \pm}=\nabla p$. However, the function $p$ only satisfies conditions (3.15), so that the displacement fields may fail to belong to the space $W_{\infty}^{1}\left(\Omega, R^{m}\right)$. This observation shows that Theorem 4.1 is not sharp.

If the temperatures $t_{ \pm}$exist, then the necessary condition for their existence implies that the numbers

$$
\begin{aligned}
& \hat{t}^{+}=-\underset{x \in \Omega}{\operatorname{essinf}}\left\{F^{+}\left(\nabla \hat{u}^{-}(x)\right)-F^{-}\left(\nabla \widehat{u}^{-}(x)\right)\right\}, \\
& \hat{t}_{-}=-\operatorname{ess}_{x \in \Omega}\left\{F^{+}\left(\nabla \widehat{u}^{+}(x)\right)-F^{-}\left(\nabla \widehat{u}^{+}(x)\right)\right\}
\end{aligned}
$$

are finite and $t_{+} \geq \hat{t}_{+}, t_{-} \leq \hat{t}_{-}$. For the problems treated in $\S 3$,

$$
F^{+}\left(\nabla \widehat{u}^{ \pm}\right)-F^{-}\left(\nabla \widehat{u}^{ \pm}\right)=m\left[a c^{2}\right]-2 \nu[a c]+\left[b(\nu-m c)^{2}\right] .
$$

Therefore, for these problems we have $\hat{t}_{-}=\hat{t}_{+}$. Consequently, generally speaking, the numbers $\hat{t}_{ \pm}$are not the temperatures of phase transitions, because, for these problems, the relation $t_{-}=t_{+}$is true only in exceptional cases (see (3.11)).

The examples in $\S 3$ show that the temperatures of phase transitions may coincide and may be different. The following statement provides sufficient conditions for the noncoincidence of the temperatures of phase transitions.

Theorem 4.2. Suppose the temperatures of phase transitions exist and $u^{+}=\widehat{u}^{-} \equiv \widehat{u}^{0}$. Then:

1) If

$$
F^{+}\left(\nabla \widehat{u}^{0}\right)-F^{-}\left(\nabla \widehat{u}^{0}\right) \neq \text { const a.e. in } \Omega
$$

then $t_{-}<t_{+}$.

2) If

$$
F^{+}\left(\nabla \widehat{u}^{0}\right)-F^{-}\left(\nabla \widehat{u}^{0}\right)=\text { const a.e. in } \Omega \text {, }
$$

but

$$
F_{M}^{+}\left(\nabla \widehat{u}^{0}\right) \neq F_{M}^{-}\left(\nabla \widehat{u}^{0}\right) \text { on a set } E \subset \Omega \text { of positive measure, }
$$

then $t_{-}<t_{+}$.

3) If condition (4.4) is satisfied and

$$
F_{M}^{+}\left(\nabla \widehat{u}^{0}\right)=F_{M}^{-}\left(\nabla \widehat{u}^{0}\right) \text { a.e. in } \Omega \text {, }
$$

then $t_{-}=t_{+}$.

If the temperatures of phase transitions exist, $\widehat{u}^{+} \not \equiv \widehat{u}^{-}$, and the inequality

$$
F^{-}\left(\nabla \hat{u}^{-}(x)\right)-F^{+}\left(\nabla \hat{u}^{-}(x)\right)>F^{-}\left(\nabla \widehat{u}^{+}(x)\right)-F^{+}\left(\nabla \widehat{u}^{+}(x)\right)
$$

is fulfilled on a set of positive measure, then $t_{-}<t_{+}$.

Proof. The proof of the first part of the theorem was given in [10]. The second part is a consequence of the necessary minimum condition (4.3).

\section{REFERENCES}

[1] M. A. Grinfel'd, Methods of continuum mechanics in the theory of phase transitions, Nauka, Moscow, 1990. (Russian) MR1128092 (93c:73012)

[2] L. C. Evans and R. F. Gariepy, Measure theory and fine properties of functions, CRC Press, Boca Raton, FL, 1992. MR,1158660 (93f:28001)

[3] V. G. Osmolovskiǔ, An existence theorem and the weak form of Lagrange equations for a variational problem in the theory of phase transformations, Sibirsk. Mat. Zh. 35 (1994), no. 4, 835-846; English transl., Siberian Math. J. 35 (1994), no. 4, 743-753. MR1302437(95h:49004)

[4] _ Phase transition in the mechanics of continuous media for big loading, Math. Nachr. 177 (1996), 233-250. MR1374951(97i:73012) 
[5] L. C. Evans, Weak convergence methods for nonlinear partial differential equations, CBMS Regional Conf. Ser. in Math., vol. 74, Amer. Math. Soc., Providence, RI, 1990. MR.1034481 (91a:35009)

[6] V. G. Osmolovski1, Criterion for the lower semicontinuity of the energy functional of a two-phase elastic medium, Probl. Mat. Anal., No. 26, Tamara Rozhkovskaya, Novosibirsk, 2003, pp. 215-254; English transl., J. Math. Sci. (N. Y.) 117 (2003), no. 3, 4211-4236. MR2027456 (2004m:74056)

[7] S. Müller, Microstructures, phase transitions and geometry, Preprint no. 3, Max-Planck-Inst. Math. in Naturwiss., Leipzig, 1997. MR1645820 (2000d:74003)

[8] V. G. Osmolovskiı̌, On the phase transition temperature in a variational problem of elasticity theory for two-phase media, Probl. Mat. Anal., No. 41, Tamara Rozhkovskaya, Novosibirsk, 2009. pp. 3747; English transl., J. Math. Sci. (N. Y.) 159 (2009), no. 2, 168-179. MR2544035 (2010k:35492)

[9] __ Exact solutions of the variational problem of phase transition theory in continuum mechanics, Probl. Mat. Anal., No. 27, Tamara Rozhkovskaya, Novosibirsk, 2004, pp. 171-205; English transl., J. Math. Sci. (N. Y.) 120 (2004), no. 2, 1167-1190. MR2099066 (2005f:74038)

[10] __ Existence of phase transitions temparatures of a nonhomogeneous anisotropic two-phase elastic medium, Probl. Mat. Anal., No. 31, Tamara Rozhkovskaya, Novosibirsk, 2005, pp. 59-66; English transl., J. Math. Sci. (N. Y.) 132 (2006), no. 4, 441-450. MR2197338 (2006k:49031)

Department of Mathematics and Mechanics, St. Petersburg State University, UniverSitetskaya Ul. 28, Stary Petergof, St. Petersburg 198504, Russia

E-mail address: vicos@V08167.spb.edu

Received 30/JUN/2010

Translated by N. B. LEBEDINSKAYA 